\title{
A Differential Planetary Gear Model with Backlash and Teeth Damage
}

\author{
Xi Wu, Jim Meagher, Andrew Sommer
}

\begin{abstract}
Planetary gear trains can be more compact and efficient as power transmissions than fixed axis gear trains but are also more complicated and less understood in terms of vibration health monitoring. A practical differential planetary gear train, which combines two inputs and one output, is studied using multi-body dynamics software. Backlash between the sun gear and planet gears are precisely specified to avoid teeth interference and undercut. In order to calculate accurate impact forces, an impact model is chosen. Tooth geometry errors are created on the sun gear. Constraints and contact forces to the model are applied as close as possible to real operating conditions. Torsional vibration induced by backlash and tooth geometry errors is shown to cause teeth separation and doublesided impacts in unloaded and lightly loaded gearing drives. Planetary gears with only backlash errors are compared to those containing both backlash and tooth defects under different kinematic and loading conditions. Time domain results show that the dynamic responses due to the combination of backlash and tooth defects depend on the interaction of many components of the differential planetary system.
\end{abstract}

\section{Introduction}

The planetary gear train is widely used in transmission design of automobiles, helicopters and aircraft engines due to the numerous advantages over traditional fixed-axis gear transmissions. Since the load being transmitted is shared between several planets, torque capability is significantly increased. One notable advantage of a planetary gear is its distinctive combination of both compactness and magnificent power transmission efficiencies. Despite the above advantages, however, the complicated dynamic forces existing among sun, planet and ring gears are difficult to analyze for the main sources of the vibration. Reasonably simulating the practical dynamic force between single-mating-gear pair still remains as an important topic. Needless to say, it is even more challenging to realistically model a planetary gear train with multiple meshing stages. As a result, the dynamic analyses of planetary gear trains have received far less attention than fixed-axis gear trains.

The earliest review papers that discuss the numerical modeling of gear dynamics focus on spur gears: Özgüven and Houser in 1988 [1] and by Parey and Tandon in 2003 [2]. The models had a limited number of degrees of freedom and most commonly did not include tooth defects. Tooth meshing stiffness was characterized as either an average or piecewise linear variation instead of the highly nonlinear Hertzian contact. Parey and Tandon's review did include spur gear defects. Parey etc. [3] developed a six DOF nonlinear model for a pair of spur gears on two shafts, calculated the Hertzian stiffness for the tooth surface contact, and implemented the empirical mode decomposition (EMD) method to simulate the different defect widths. The above research is based on fix-axis gears.

Lin and Parker [4] analytically investigate the parametric instability of planetary gears induced by gear mesh stiffness variation. The authors use rectangular waveforms with different contact ratios and mesh phasing to simulate the gear mesh stiffnesses existing between sun-planet and planet-ring gear mating pairs. Instability boundaries are directly associated with meshing parameters in the vibration modes. The authors also demonstrate some numerical simulation results about the teeth separation caused by parametric instability and strong impact in the system response. Lin and Parker [5] derive a theoretical model and carefully identify the important characteristics of the natural frequencies and vibration modes for planetary gears. The model uses three planar degrees of freedom for each component of the planetary gears and takes gyroscopic effects and time-varying gear mesh stiffnesses into consideration. The authors do comprehensive investigations about the distinctive characteristics of each type of mode. Based on the model of reference [5], Guo and Parker [6] extended the twodimensional lumped-parameter model by incorporating the factors of teeth separation, back-side contact, tooth wedging, and bearing clearances. By scrutinizing the dynamic response of an example planetary gear, the authors investigate the nonlinear tooth wedging behavior often observed on a wind turbine planetary gear train. More 
research about dynamic behavior of planetary gears can be found in reference [7, 8]. Unfortunately, the majority of current papers about planetary gears does not include the interactive effects of backlash and gear teeth damage.

Although simplified model for even a one-stage gear train involves sophisticated mathematics, it cannot accurately simulate the gear train's practical dynamic behavior even for an ideal system by simply assuming timevarying gear mesh stiffness as square waveforms. The impact forces between the mating pairs are very sensitive to the geometric profile of gear tooth and the gear backlash which must be carefully designed. Geometric simplifications can be overcome by combining CAD models with ADAMS. Kong and Meagher etc. [9] modeled the nonlinear contact mechanics of large gearbox in that way but backlash was not included.

In this study, a practical differential planetary gear train, which combines two inputs and one output, is studied using multi-body dynamics software. The backlash between the sun gear and planet gears are carefully designed and calculated to avoid teeth interference and undercut. Tooth profile errors are introduced for comparison to ideal gears. The nonlinear contact mechanics model of the meshing teeth is built by careful calculation and selection of the contact simulation parameters such as the stiffness, force exponent, and damping and friction coefficients. Planetary gears with only backlash errors are compared to those containing both backlash and tooth defects under different kinematic and loading conditions. Time domain results will show that the dynamic responses due to the combination of backlash and tooth defects depend on the interaction of many components of the differential planetary system.

\section{Modeling a differential planetary gear train with backlash and teeth damage}

The differential planetary gear chosen for study has two inputs and one output $[11,12,13]$. The transmission schematic and CAD model are shown in Fig. 1 (a), and Fig. 1 (b), respectively. The profile of the "chipped" sun gear tooth in shown in Fig.2. Geometric parameters of the planetary gear train are shown in Table 1.

Combined with gear profile errors, the backlash may cause the loss of contact between gear teeth. This may induce large impact forces associated with consecutive single-sided and/or double-sided impacts. The contact surfaces between the gears are modeled as deformable flex-bodies. The nonlinear contact force, $=\mathrm{K}(\mathrm{d})^{\mathrm{e}}-c v$, is composed of an elastic and damping portion [10]. $d$ is the penetration depth. The damping force, $c v$, is proportional to impact velocity, $v$. The stiffness coefficient, $K$, is taken to be the average value of stiffness over one tooth mesh cycle. The force exponent, e, was determined from trial simulations. The damping coefficient generally takes a numeric value between $0.1 \%-1 \%$ of $K$. The determination of force exponents however is not obvious and must be based on experience.

The MSC.ADAMS IMPACT algorithm was chosen as the contact force model because of its robustness in numerical integration. The restitution model is extremely sensitive to the duration of the contact event, and is best suited for impulse type simulations. It is not ideal for time histories that include a large number of contact events in which the force vector is not known beforehand. The stiffness parameter is reasonable for this lightly loaded steel gear pair, and was determined via a trial and error method. The response of interest occurs over a very short time interval, around one hundred milliseconds. Because the damping force in meshing gears is such a small percentage of $K$, its affect on the simulation results is not significant. Therefore, the damping coefficient is kept as near to zero as possible to simplify the numerical solver routine. Penetration depth is defined here as the depth at which the damping force becomes active. Similarly, modification of this value does not have a significant effect on response of either gear. The MSC.ADAMS contact parameters are shown in Table 1. For ease of comparison, the dynamic conditions of each figure are shown in Table 2.

\section{Dynamic response and discussion of a planetary gear with the ring gear fixed}

When ring gear $\mathrm{b}$ and gear 1 are fixed, an initial angular velocity $\omega_{x 0}=100 \mathrm{rad} / \mathrm{sec}$ is applied on the carrier $\mathrm{x}$. The carrier and sun gear are rotating in the same direction. By inspection of Fig.3, the planets must be rotating in the opposite direction of both the sun and carrier. The responses of the planet gears are nearly identical. This is expected because their axes are moving with the rotating carrier, and therefore make contact with both the sun and ring at nearly the same instant. 
Consider the situation in Fig.4 when two or more planets experience nearly identical forces. This occurs when they are both in contact with a given element, either the sun or ring, and are "sharing" the load. Each planet gear is given an arbitrary designation of either 1,2 , or 3 , respectively. Between 5 and $15 \mathrm{~ms}$ planet 1 is the only planet in contact with the sun, therefore both planet 1 and planet 3 are traveling within their respective backlash. During this same interval, planet1 is not in contact with the ring. The MSC.ADAMS simulation model includes six contact forces: three on the sun gear from each planet, and three on the ring gear from each planet. At any instant in time, no force or any combination of all six forces are potentially active. Due to the small damping prescribed in the contact force, this pattern will completely die out after a significant amount of time has passed. The pattern enclosed shown between 0 and $30 \mathrm{~ms}$ continuously repeats with slightly less magnitude on each repetition.

A near equilibrium position is utilized in all presented simulations. First, consider the situation when the transmission is in an exactly symmetrical position. The front and right planes of the sun and ring would be coincident, and their top planes parallel. Each of the three planets would be in the exact center of the backlash space of the sun and ring meshes. In this position the response of each planet gear would be identical, and contact would occur at the exact same instant. The symmetrical position is deliberately avoided. The sun, planets, and ring begin within the backlash space, but slightly displaced from the perfect center. In this way contact occurs on each element at different times, and the interesting force response of Fig.4 are observed. This near equilibrium position creates the difference in contact force magnitudes, which is driving the behavior of the planetary transmission. The near equilibrium position is utilized in all simulations presented in this investigation.

\section{Dynamic response and discussion of a planetary gear with free ring gear}

This discussion is an extension of the free vibration explored in section 3. Both the ring gear and gear 1 are now unconstrained and allowed to rotate freely. The initial velocity of the carrier is now transferred into all of the transmission elements, instead of only the sun and planets. Due to the ring gear's relatively large mass moment of inertia, the introduction of its rotation causes some interesting phenomena.

The results of this more complicated free vibration are counter-intuitive. The elements no longer oscillate with the same frequency with respect to the equilibrium position. The system returns to this position at $93 \mathrm{~ms}$, instead of the quicker period of 20ms shown in Fig.3. The sun, planets, and carrier have the same period of $27 \mathrm{~ms}$ because their rotations are coupled together. Similarly, a rotation of gear1 must cause a rotation of the ring gear, and they are coupled together with the longer period of $93 \mathrm{~ms}$. Fig. 5 depicts the relatively large system period of $93 \mathrm{~ms}$.

Significantly more vibration occurs when an element reverses direction, shown in Fig.6. This is because of the "time lag" caused by the various interplay of inertias. In section 3 when the sun makes contact with a planet, the planet in turn makes contact with the ring which is a fixed rigid body. In this configuration when the sun makes contact with a planet, the planet in turn makes contact with the ring, which in turn makes contact with gear 1, which is freely rotating. Because no fixed element is present, only the gear inertias are contributing to the change in angular velocity. Between 25 and $35 \mathrm{~ms}$ the sun makes contact with various planets, and its inertia makes a more significant change in the planet's rotation than if the ring was fixed. The end result is this larger "settling time" as the sun reaches its new angular velocity at $35 \mathrm{~ms}$.

The magnitude of the contact force between dynamic elements is shown in Fig.7. A comparison with the analogous plot shown in Fig.4 yields some important conclusions. The repeating symmetry of section 3 is not observed. The first significant impact, occurs faster than it does in the fixed ring configuration. The interval in which the impact takes place has decreased. For example, the sun and planet 2 are in contact for $20 \mathrm{~ms}$ in section 3, but now only remain in contact for $15 \mathrm{~ms}$. This can be explained by again considering that the inertias of all components are now interacting. When the sun makes contact with planet 2 the rotation of the ring and gear1 are ultimately effected, and therefore the time in which these two elements are in contact is less than if the ring and gearl were fixed. The general shape of the curve is the same, only the impact duration has changed.

\section{Dynamic response and discussion of a planetary gear with constant velocity from sun gear}

The ring gear is fixed and the sun gear is being driven by Motor 1, which is assumed to have a constant angular speed of $975 \mathrm{rpm}=102.1 \mathrm{rad} / \mathrm{sec}$. Fig. 8 is the magnitude of each gear's angular velocity. Obviously, the 
planet gears must be rotating in a direction opposite that of the sun and carrier. For the first few milliseconds it is observed that the angular velocities change rapidly, and converge to an average speed based on the gear ratios. The planets have a much smaller rotary inertia relative to the carrier assembly. Since the planets experience forces from the sun and internal ring, it follows that their angular velocities should change with a larger magnitude. These "oscillations" die out rather quickly because the system is being driven by a kinematic motion constraint. In other words, the sun will have a constant velocity regardless of any force it experiences. This loading assumption essentially "forces" the other elements to reach steady-state very quickly.

The sun and planet 1 share an interesting force history shown in Fig.9. The large peaks can be attributed to the configuration in which the sun is in contact with only one planet. The smaller peaks include "load sharing" between the other two planet gears. The force between the sun this planet is reduced when the sun is in contact with the other planet gears. The peaks and troughs of this plot are due to the rapidly changing contact ratio on the sun.

Consideration of a sun gear with a "chipped" tooth is depicted in Fig.10. This planet gear is in mesh with the chipped area of the sun. In the first millisecond, fewer impacts have occurred because of the damaged tooth. It takes longer for the gears to make contact because geometry is simply missing. The magnitude of the velocity change has also increased, particularly between 1 and $3 \mathrm{~ms}$. This is because the planet gear has more room to move in the backlash due to the gap created by the missing tooth. An impact causes a change in acceleration, which is allowed to propagate further because of the gap. The response of the gears become nearly identical after $10 \mathrm{~ms}$ because the chipped tooth has moved through the mesh.

\section{Dynamic response and discussion of a planetary gear with a step torque applied to the sun gear} With the ring gear fixed, a step input torque with the magnitude of $70.518 \mathrm{~N}-\mathrm{m}$ is applied on the sun gear over 100 ms. A step torque of the form $\mathrm{T}\left(1-e^{t / \tau}\right)$ is applied to represent a typical electric motor characteristic curve. The torque magnitude and time constant is calculated using the rated torque and rated speed.

Compare the contact force magnitude of Fig. 11 with the analogous plot of Fig.9. The first significantly large magnitude response occurs at $9 \mathrm{~ms}$ for the constant input speed section 5, while the same magnitude here begins at $25 \mathrm{~ms}$. This delay is due the dynamic nature of torque. A torque applied to a body will not instantaneously cause motion, instead it causes an angular acceleration. This acceleration works against the inertia of the sun, causing the response to occur later than the constant speed case. The smaller peaks and troughs are again due to the rapidly changing contact ratio on the sun. The largest peaks occur when this planet is the only planet gear in contact with the sun.

The chipped sun experiences fewer impact events than the standard sun, shown in Fig. 12. This is because an entire tooth is missing and it takes longer for the gears to contact. The magnitude of the velocity changes for the chipped sun are larger. This is because the torque has had more time to accelerate the sun before contact is made. This larger velocity change corresponds to a larger force magnitude. The chipped tooth causes the sun to experience more harsh start-up conditions. These oscillations die out quickly as the torque continues to accelerate the system, and the contact between gear teeth becomes constant on one side only. Velocity changes from positive to negative, like those shown here, represent double sided impacts.

\section{Dynamic response and discussion of a planetary gear with a step torque applied to both sun gear and gear} 1

The system is operating as a differential transmission, with both Motor 1 and Motor 2 in operating in the same direction. A step torque of the form $T\left(1-\mathrm{e}^{\mathrm{t} / \tau}\right)$ is applied to represent a typical electric motor characteristic curve. The torque magnitude and time constant is calculated using the rated torque and rated speed, which are $70.518 \mathrm{~N}-\mathrm{m}$ and $67.935 \mathrm{~N}-\mathrm{m}$ on the sun and gear 1, respectively.

With the ring gear fixed as in Fig.13(a), the sun gear closely follows the input torque curve. The planets dissipate energy from the sun input to the carrier output. The planets must get the carrier inertia moving from rest. The sun, having the smallest inertia, oscillates with the largest amplitude of any gear. The amplitude of the sun 
gear's velocity change is decreased with the system operating as a differential transmission, shown in Fig.13(b). The rotation of the ring causes the planets to move in the same direction as the applied torque on the sun. This makes the angular velocity of the sun more smooth when compared to the simple planetary configuration of the fixed ring. This effect is also seen in the other gears. The detrimental effects of the chipped tooth on the sun gear is lessened with the system operating in the differential mode.

The gear 1 and ring mesh has a smaller prescribed backlash that the meshes between the sun and planets. Therefore, contact occurs between these two gears before the sun and planets. A clear succession of contacts is observed. First gear1 contacts the ring, the ring contacts a planet, and the planet contacts the sun. The interval from $3.5 \mathrm{~ms}$ to $4.5 \mathrm{~ms}$ in Fig. 14 clearly shows this progression. The planet gears are potentially in contact with both the sun and ring. The contact between gear 1 and the ring directly affect the magnitude of the force between the planet and ring, shown as the smaller peak at $7.5 \mathrm{~ms}$. The response of planet gear is a superposition of impact forces between its sun mesh and ring mesh.

\section{Conclusions}

A practical differential planetary gear train which combines two inputs and one output has been analyzed using a non-linear multi-body dynamics software model. To avoid interference and undercut the backlash between the sun/planet and planet/ring meshes are precisely specified. When the transmission operates with a fixed ring gear and undergoes free vibration from a near equilibrium position, six contact forces are potentially active. The magnitude of the contact force between each element is shown to depend on the rapidly changing contact ratios of each gear. For free vibration and a freely rotating ring, the duration of the contact event between gears decreases. When compared to the perfect mesh geometry, a planet in mesh with the chipped tooth of the sun causes its angular velocity to change with a larger magnitude. Step torques of the form $T\left(1-e^{t / t}\right)$ applied in opposite directions to each input closely models the constraints and loading conditions of realistic operation. The transient response of this differential mode is shown to be significantly less destructive to the sun gear. The interactions of many components of this differential planetary transmission contribute to the complicated transient behavior observed in the time domain.

\section{Acknowledgement}

The authors acknowledge the Donald E. Bently Center for Engineering Innovation at California Polytechnic State University San Luis Obispo for support of this work. 


\section{REFERENCES}

1. Özgüven H.N. and Houser D.R., "Mathematical Models used in Gear Dynamics - A Review", Journal of Sound and Vibration, 121(3), 1988, pp. 383-411.

2. Parey, A. and Tandon, N., "Spur Gear Dynamic Models Including Defects: A Review", The Shock and Vibration Digest, Vol. 35, No. 6, Nov. 2003; pp. 465-478.

3. Parey, M. El Badaoui, F. Guillet, N. Tandon, "Dynamic modeling of spur gear pair and application of empirical mode decomposition-based statistical analysis for early detection of localized tooth defect", Journal of Sound and Vibration Vol. 294, 2006, pp. 547-561.

4. J. Lin and R. G. Parker, "Parametric Instability of Planetary Gears under Mesh Stiffness Variation", Journal of Sound and Vibration, 2002, vol. 249, pp. 129-145.

5. J. Lin and R. G. Parker, "Analytical Characterization of the Unique Properties of Planetary Gear Free Vibration”, ASME Journal of Vibration and Acoustics, vol. 121, July 1999, pp. 316-321.

6. Yi Guo and R. G. Parker, "Dynamic Modeling and Analysis of a Spur Planetary Gear Involving Tooth Wedging and Bearing Clearance Nonlinearity", European Journal of Mechanics A/Solids, 2010, vol. 29, pp. 1022-1033.

7. R. G. Parker and X. Wu, "Vibration Modes of Planetary Gears with Unequally Spaced Planets and an Elastic Ring Gear", Journal of Sound and Vibration, 2010, vol. 329, pp. 2265-2275.

8. Yichao Guo and R. G. Parker, "Purely Rotational Model and Vibration Modes of Compound Planetary Gears", Mechanism and Machine Theory, 2010, vol. 45, pp. 365-377.

9. Kong, D., Meagher, J., Xu, C., Wu, X., Wu, Y., "Nonlinear Contact Analysis of Gear Teeth for Malfunction Diagnostics", IMAC XXVI a Conference on Structural Dynamics, Society for Experimental Mechanics, Orlando, Florida USA, February 4 -7, 2008.

10. MSC Inc., MSC ADAMS reference manual.

11. Raymond J. Drago, "Fundamentals of Gear Design”, 1988.

12. Huamin Li, Guixian Li, "Gear Mechanism Design and Application”, 2007 (in Chinese).

13. Zengang Rao, "Planetary Gear Transmission Design”, 2003 (in Chinese). 


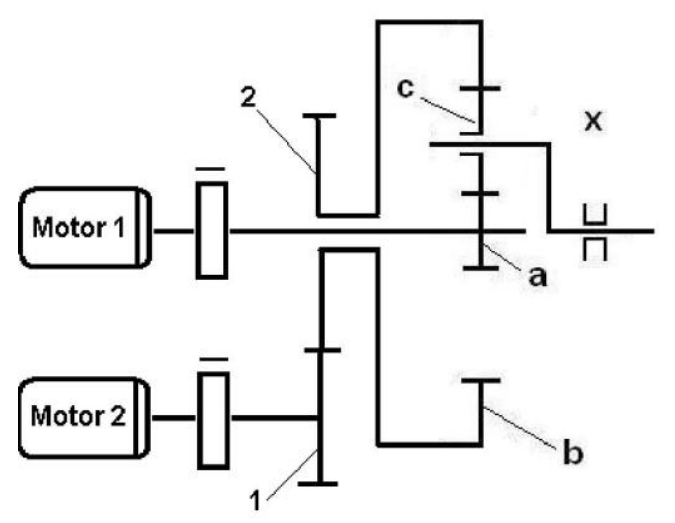

(a) Schematic

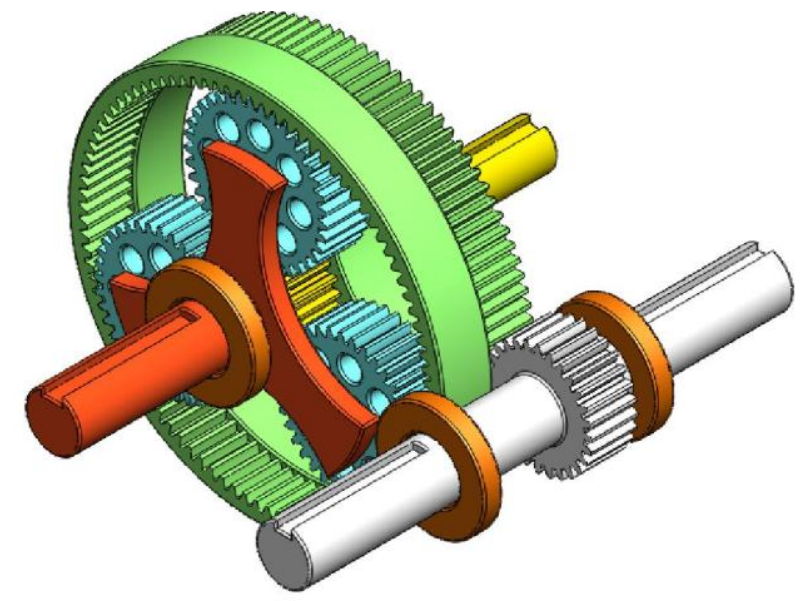

(b) CAD Isometric View

Fig.1 A differential planetary gear with two inputs and one output

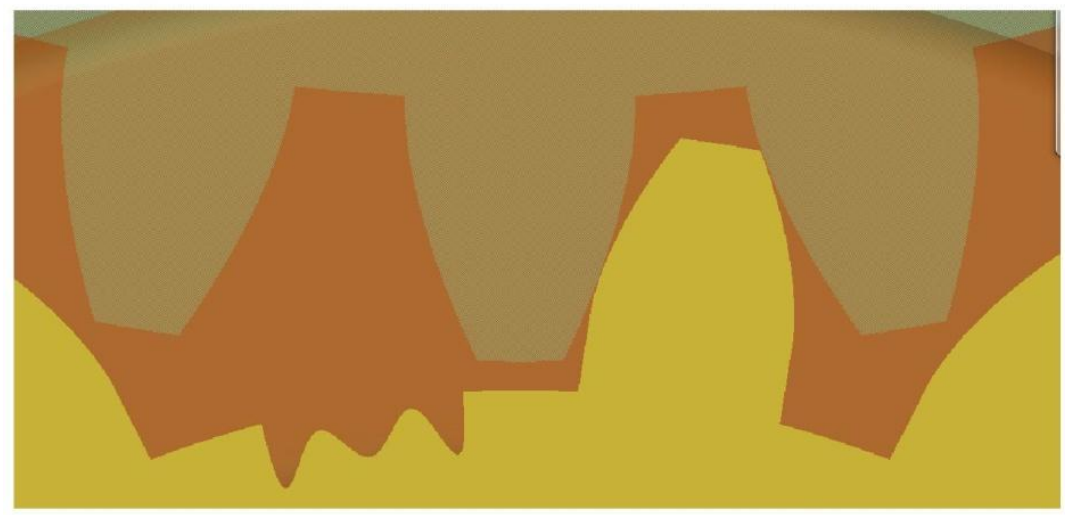

Fig.2 Chipped tooth profile 
Table 1. Planetary gear design and simulation parameters

\begin{tabular}{|c|c|c|c|}
\hline Module $\mathrm{m}(\mathrm{mm})$ & $\mathrm{m}=2$ & Backlashes $(\mathrm{mm})$ & $\mathrm{B}_{1}=0.04 ; \mathrm{B}_{2}=0.03$ \\
\hline Number of teeth & $\begin{array}{c}\mathrm{Z}_{\mathrm{a}}=20 ; \mathrm{Z}_{\mathrm{b}}=94 ; \\
\mathrm{Z}_{\mathrm{c}}=37 ; \mathrm{Z}_{1}=28 ; \mathrm{Z}_{2}=98\end{array}$ & Material properties & $\begin{array}{c}\mathrm{E}=2.07 \times 10^{11} \mathrm{~Pa} \\
v=0.29 ; \\
\rho=7801 \mathrm{~kg} / \mathrm{m}^{3}\end{array}$ \\
\hline $\begin{array}{c}\text { Standard Pitch circle } \\
\text { diameter } d(\mathrm{~mm})\end{array}$ & $\begin{array}{c}\mathrm{d}_{\mathrm{a}}=40 ; \mathrm{d}_{\mathrm{b}}=188 ; \\
\mathrm{d}_{\mathrm{c}}=74 ; \mathrm{d}_{1}=56 ; \mathrm{d}_{2}=196\end{array}$ & Force exponent & 2.2 \\
\hline Gear Ratios & $5.7 ; 1.213$ & Penetration & $10^{-7}(\mathrm{~mm})$ \\
\hline Pressure angle & $20^{\circ}$ & Stiffness & $2 \times 10^{7}(\mathrm{~N} / \mathrm{mm})$ \\
\hline
\end{tabular}

Table 2. Dynamic Conditions Depicted in Figures

\begin{tabular}{|c|c|}
\hline Fig. No. & Simulation Parameters \\
\hline $3,4,5$ & $\omega_{\mathrm{x} 0}=100 \mathrm{rad} / \mathrm{sec}$ initial angular velocity applied to the carrier, fixed ring \\
\hline 6,7 & $\omega_{\mathrm{x} 0}=100 \mathrm{rad} / \mathrm{sec}$ initial angular velocity applied to the carrier, free ring \\
\hline $8,9,10$ & $\omega_{1}=102.1 \mathrm{rad} / \mathrm{sec}$ constant angular velocity applied to the sun, fixed ring \\
\hline $11,12,13(\mathrm{a})$ & $\mathrm{T}_{1}=70.518 \mathrm{~N}-\mathrm{m}$ step torque applied to the sun, fixed ring \\
\hline $13(\mathrm{~b}), 14$ & $\mathrm{~T}_{1}=70.518 \mathrm{~N}-\mathrm{m}$ step torque applied to the sun, $\mathrm{T}_{2}=67.935 \mathrm{~N}-\mathrm{m}$ applied to gear 1 \\
\hline
\end{tabular}

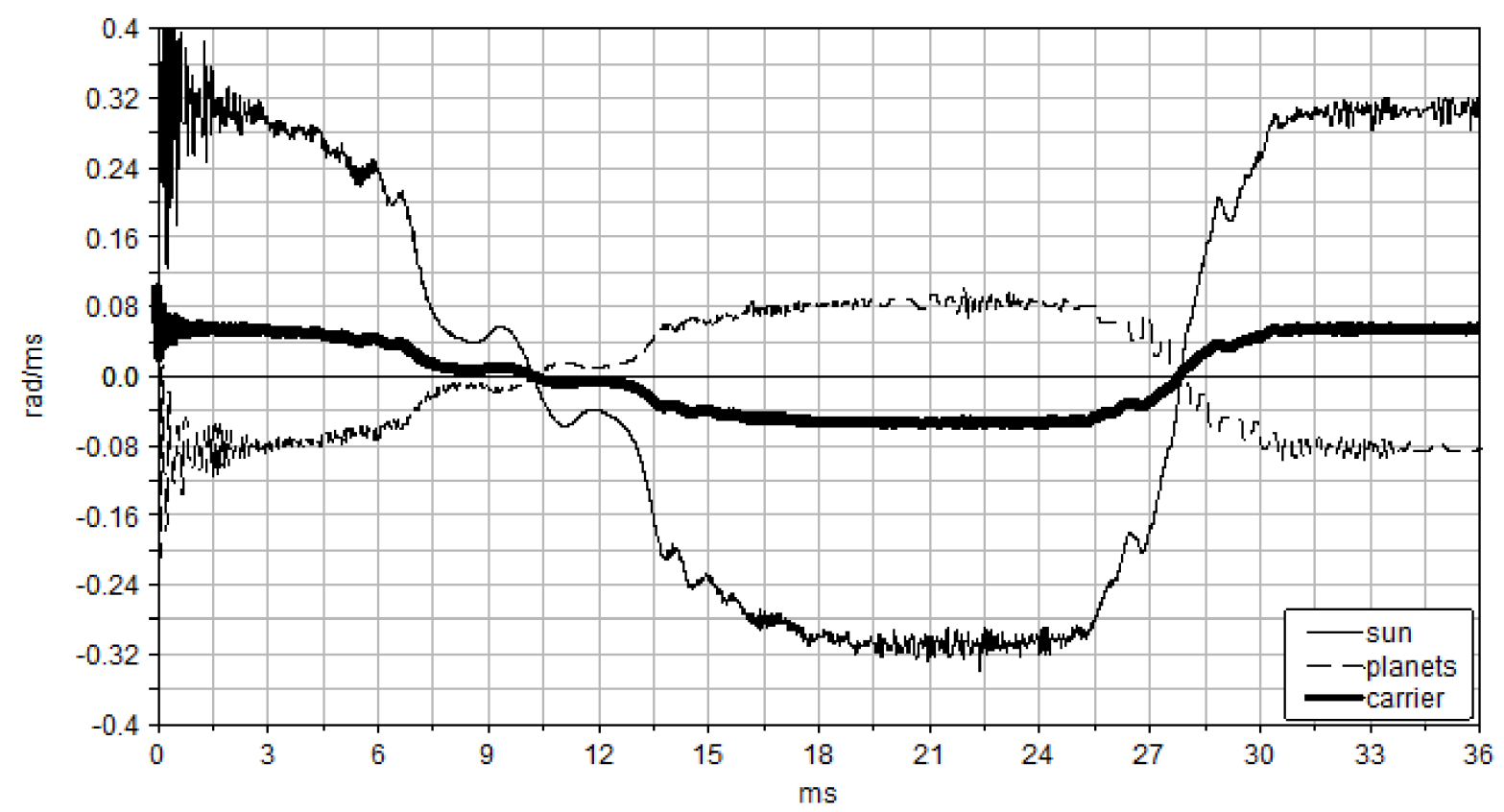

Fig.3 Angular velocity vs. time, when initial angular velocity $\omega_{x 0}=100 \mathrm{rad} / \mathrm{sec}$ is applied to the carrier 


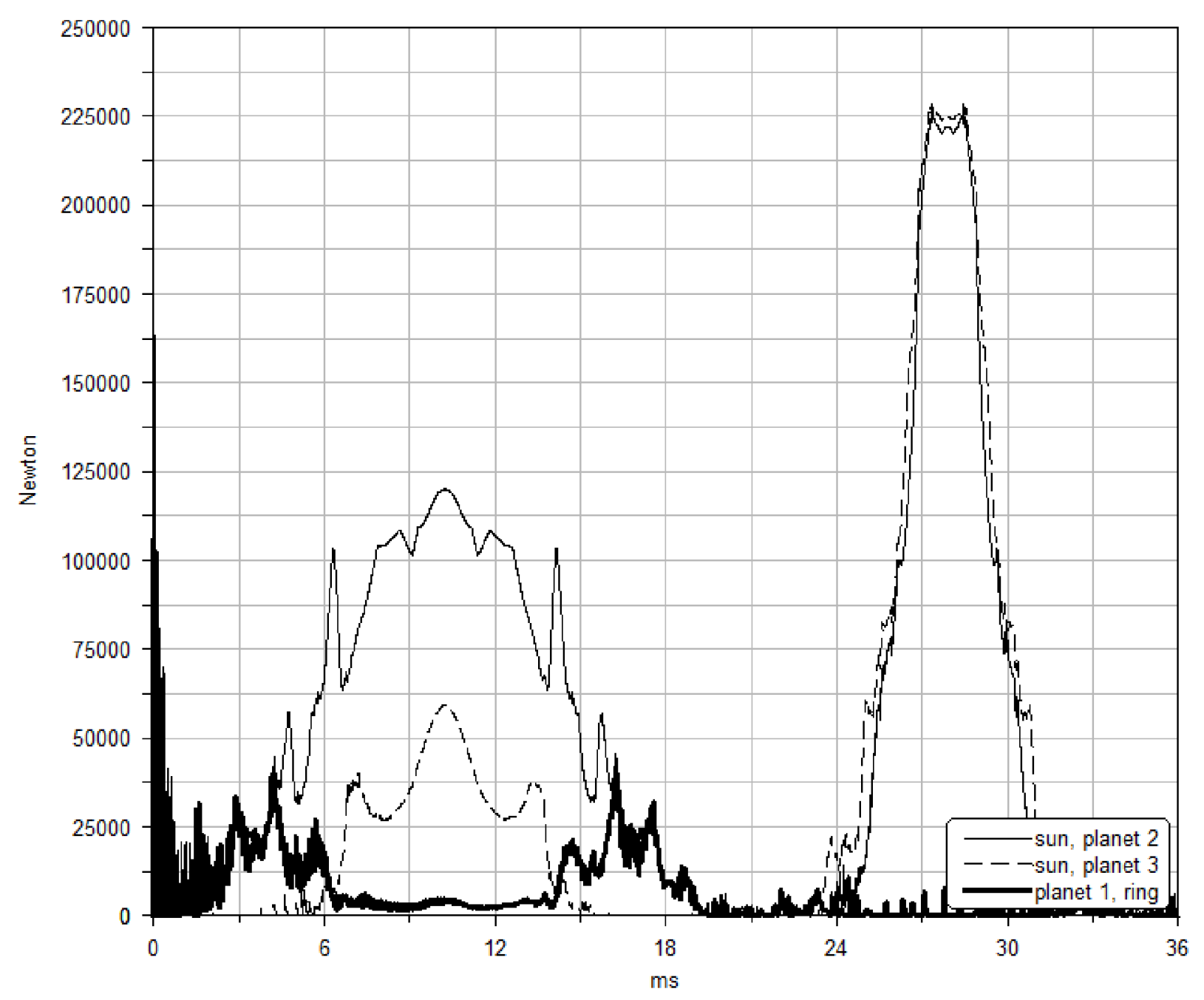

Fig.4 Contact forces vs. time, when initial angular velocity $\omega_{\mathbf{x} 0}=100 \mathrm{rad} / \mathrm{sec}$ is applied to the carrier 


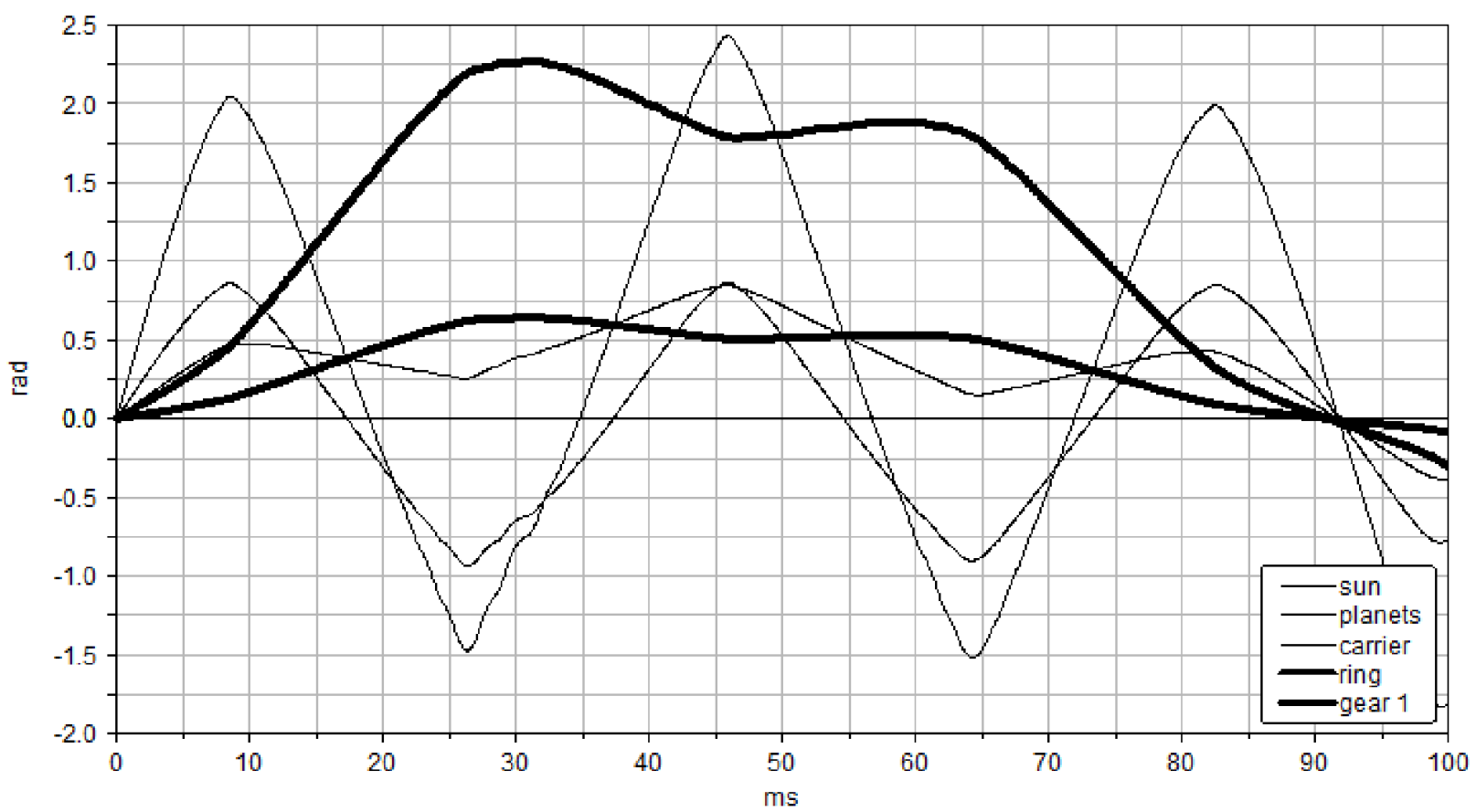

Fig.5 Angular displacement vs. time, when initial angular velocity $\omega_{\mathbf{x} 0}=100 \mathrm{rad} / \mathrm{sec}$ is applied to the carrier

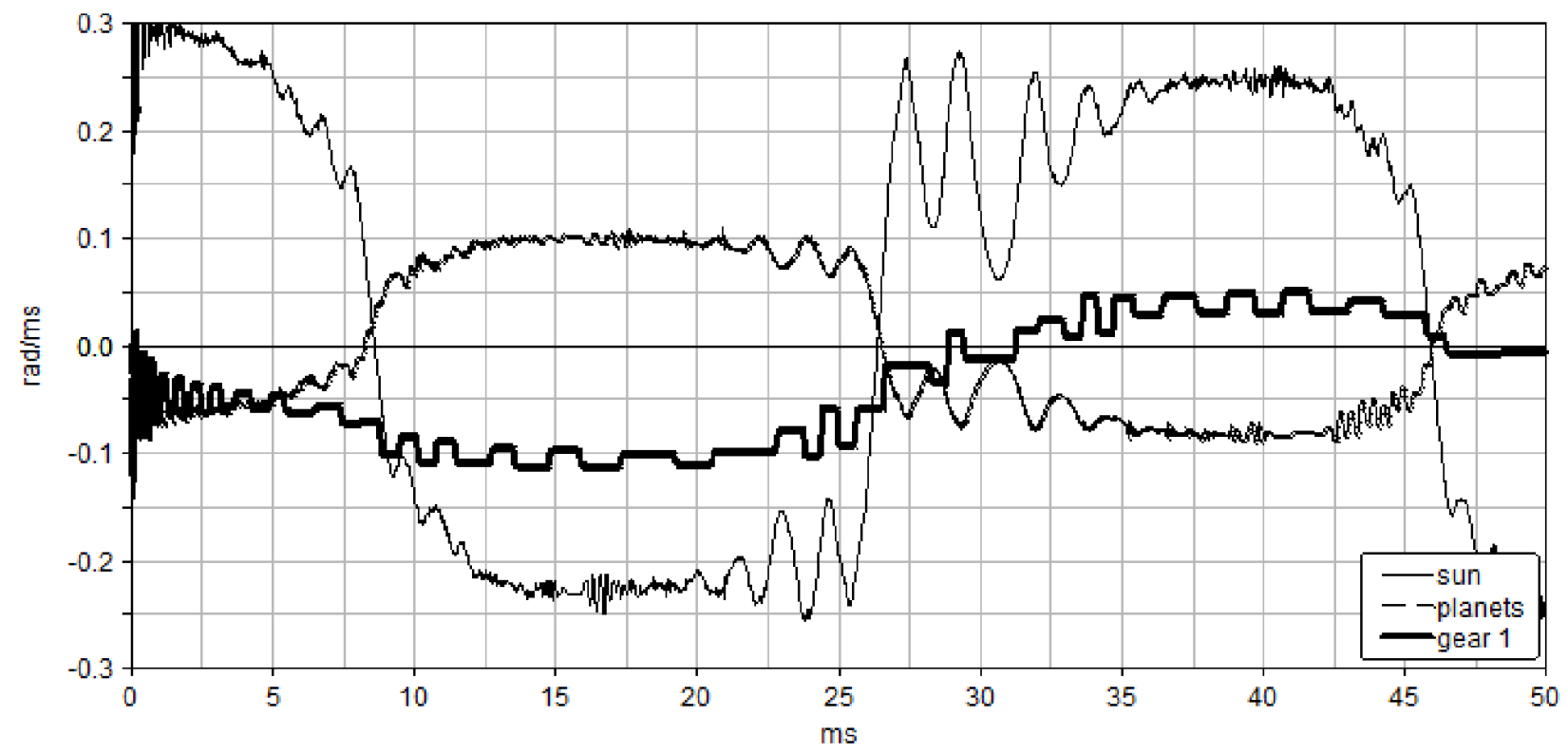

Fig.6 Angular velocity vs. time, when initial angular velocity $\omega_{\mathbf{x} 0}=100 \mathrm{rad} / \mathrm{sec}$ is applied to the carrier 


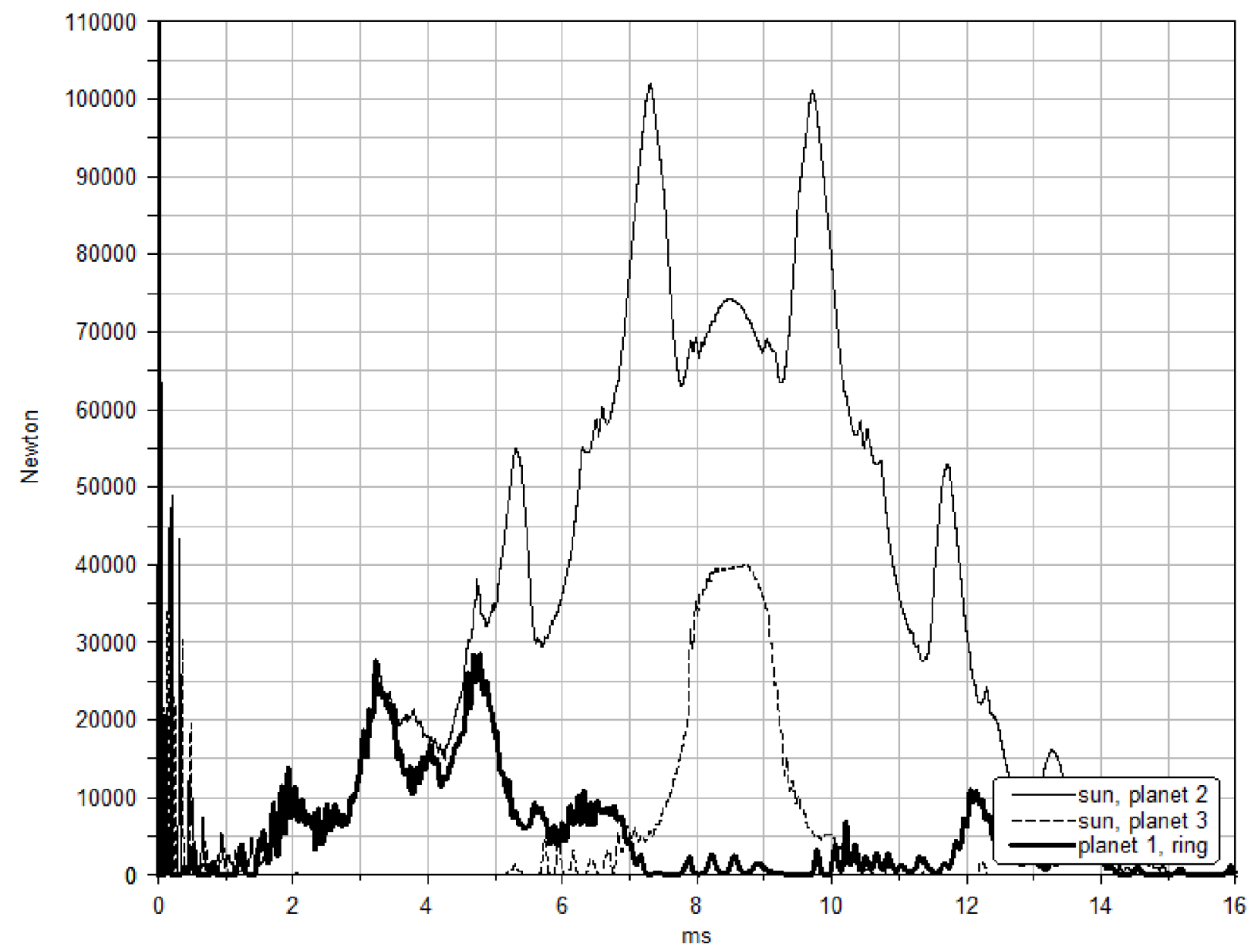

Fig. 7 Contact forces vs. time, when initial angular velocity $\omega_{\mathbf{x} 0}=100 \mathrm{rad} / \mathrm{sec}$ is applied to the carrier

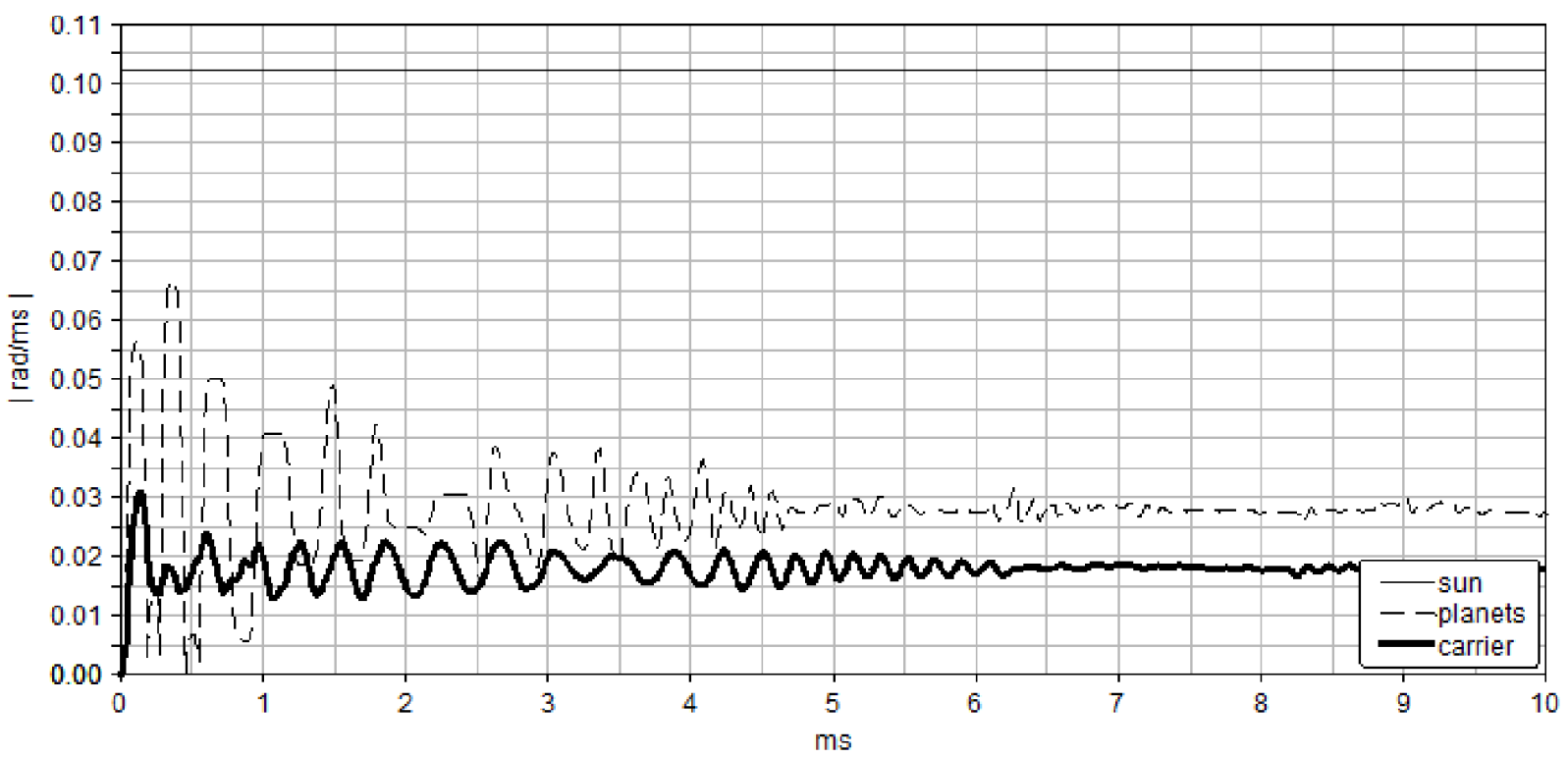

Fig.8 Angular velocities vs. time, when constant angular velocity $\omega_{1}=102.1 \mathrm{rad} / \mathrm{sec}$ is applied to the sun 


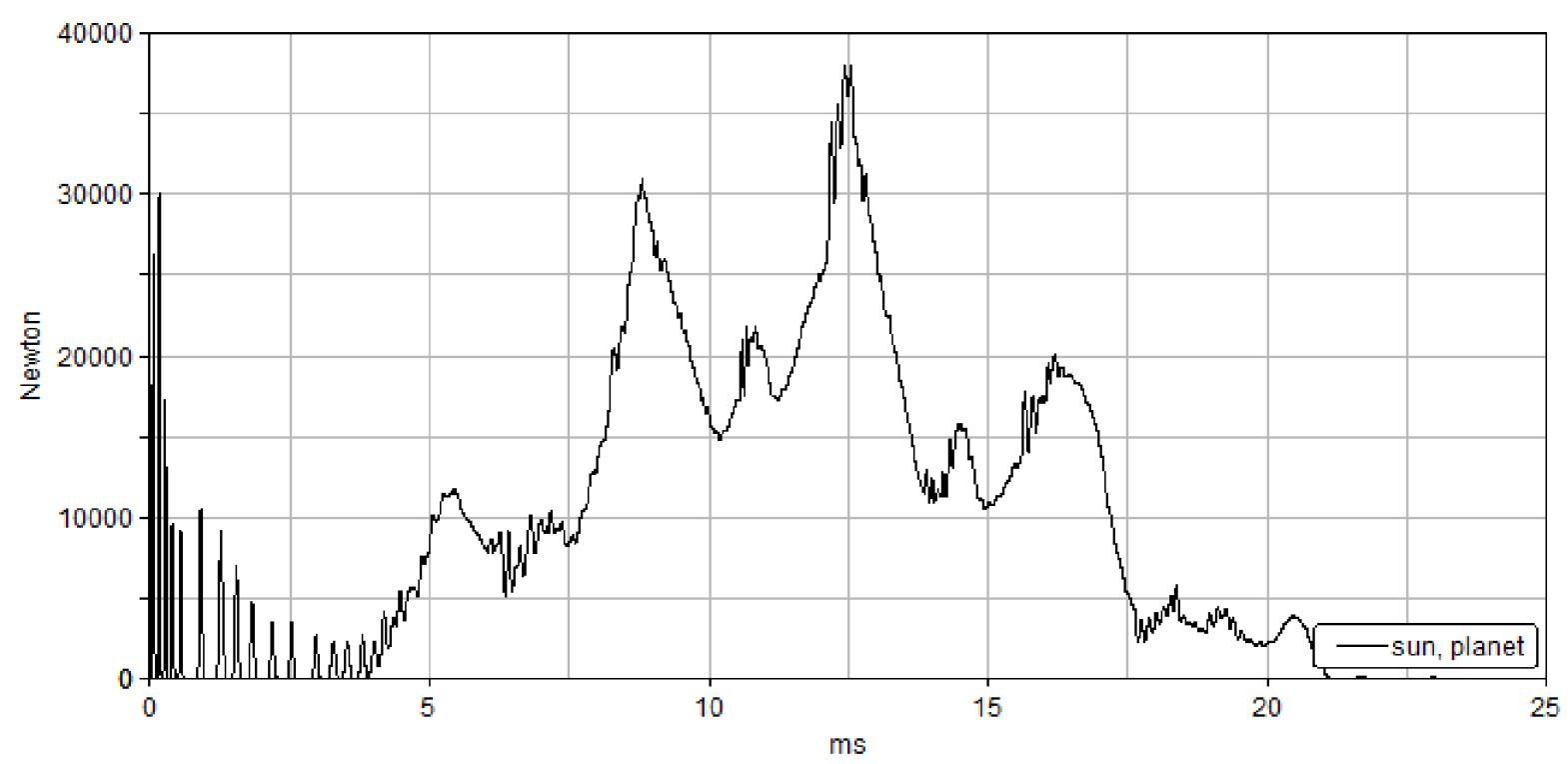

Fig.9 Sun-planet contact force vs. time, when constant angular velocity $\omega_{1}=102.1 \mathrm{rad} / \mathrm{sec}$ is applied to the sun gear

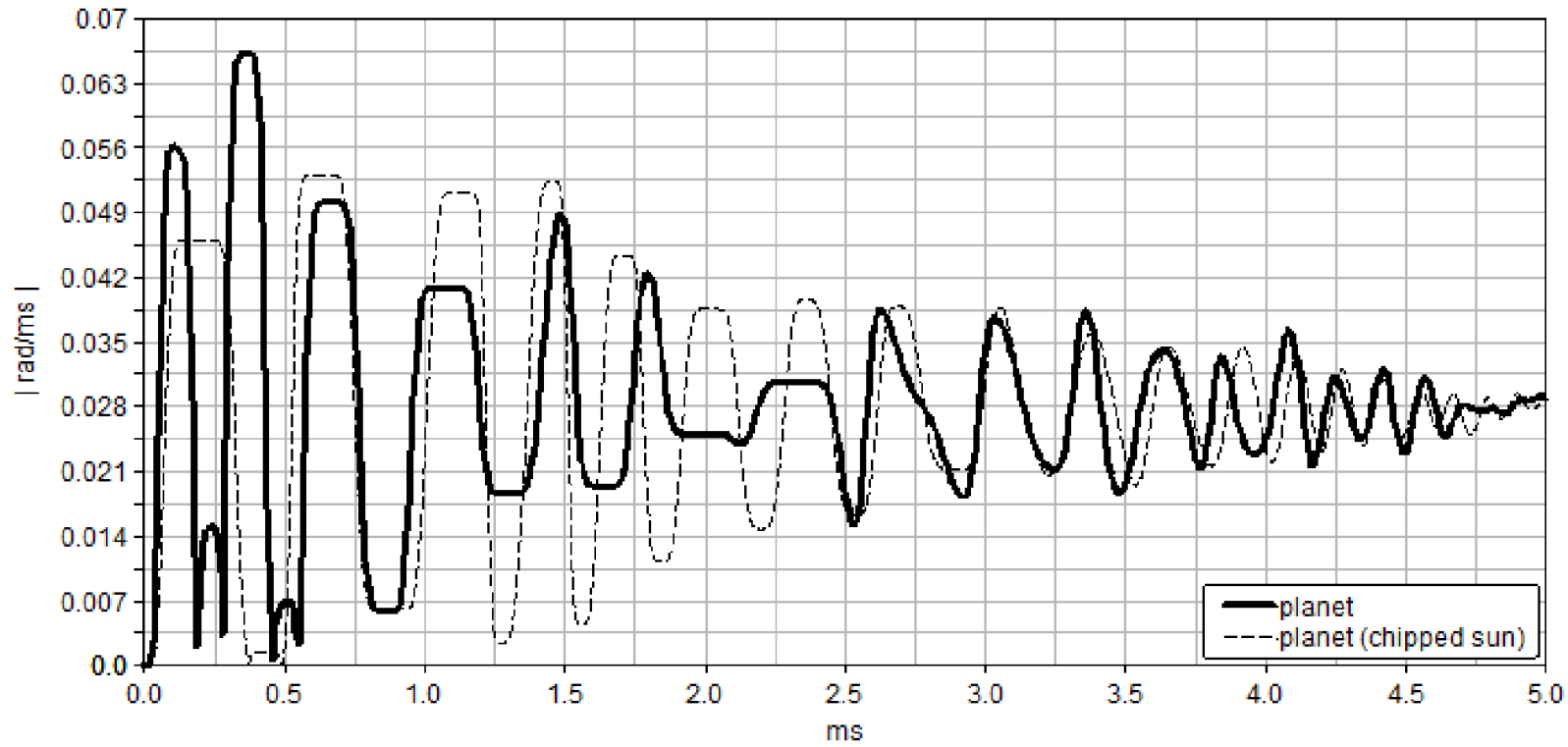

Fig.10 Angular velocities vs. time, when constant angular velocity $\omega_{1}=102.1 \mathrm{rad} / \mathrm{sec}$ is applied to the sun 


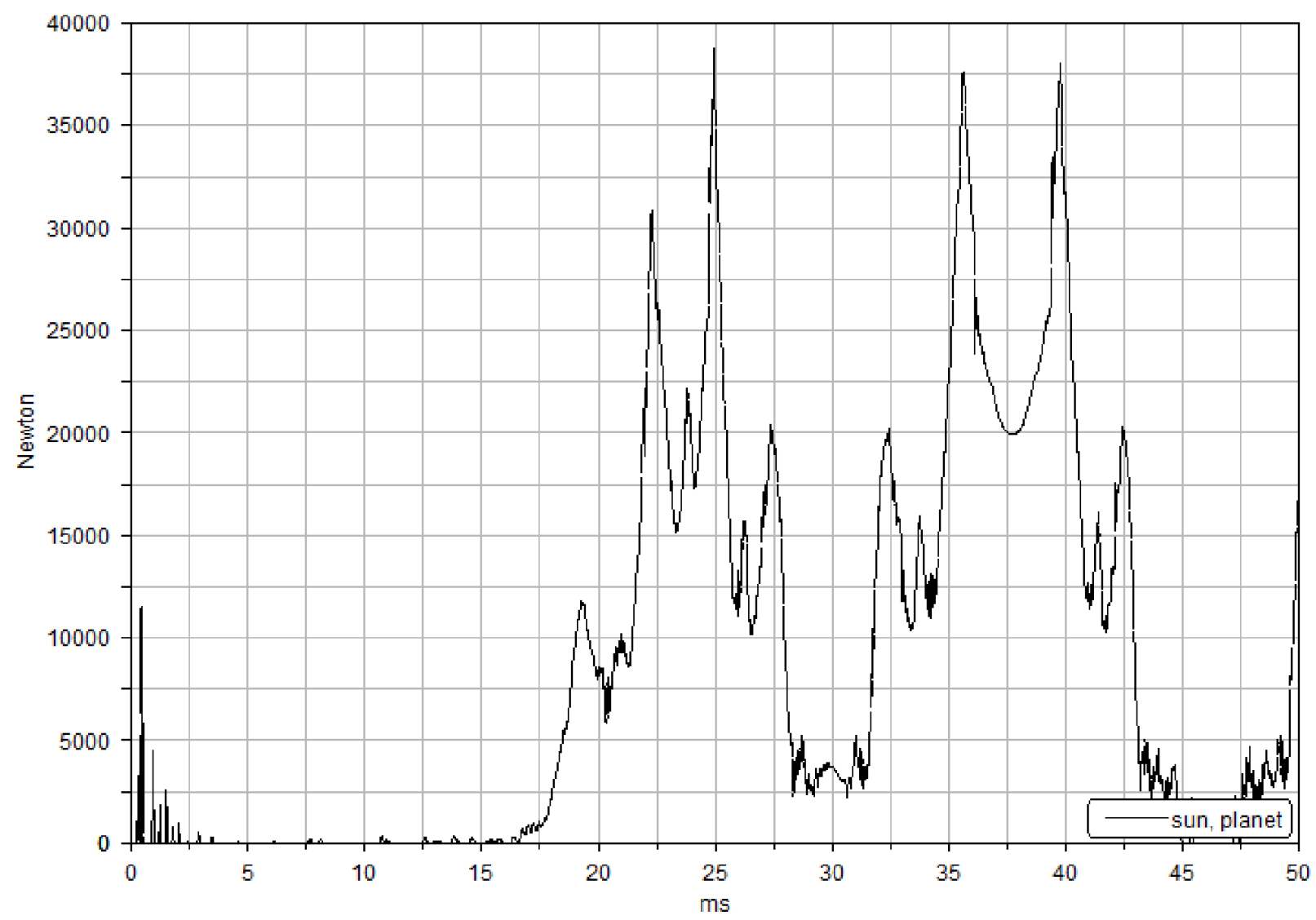

Fig.11. Sun-planet contact force vs. time, when the torque $T_{1}=70.518 \mathrm{~N}-\mathrm{m}$ is applied to the sun gear 


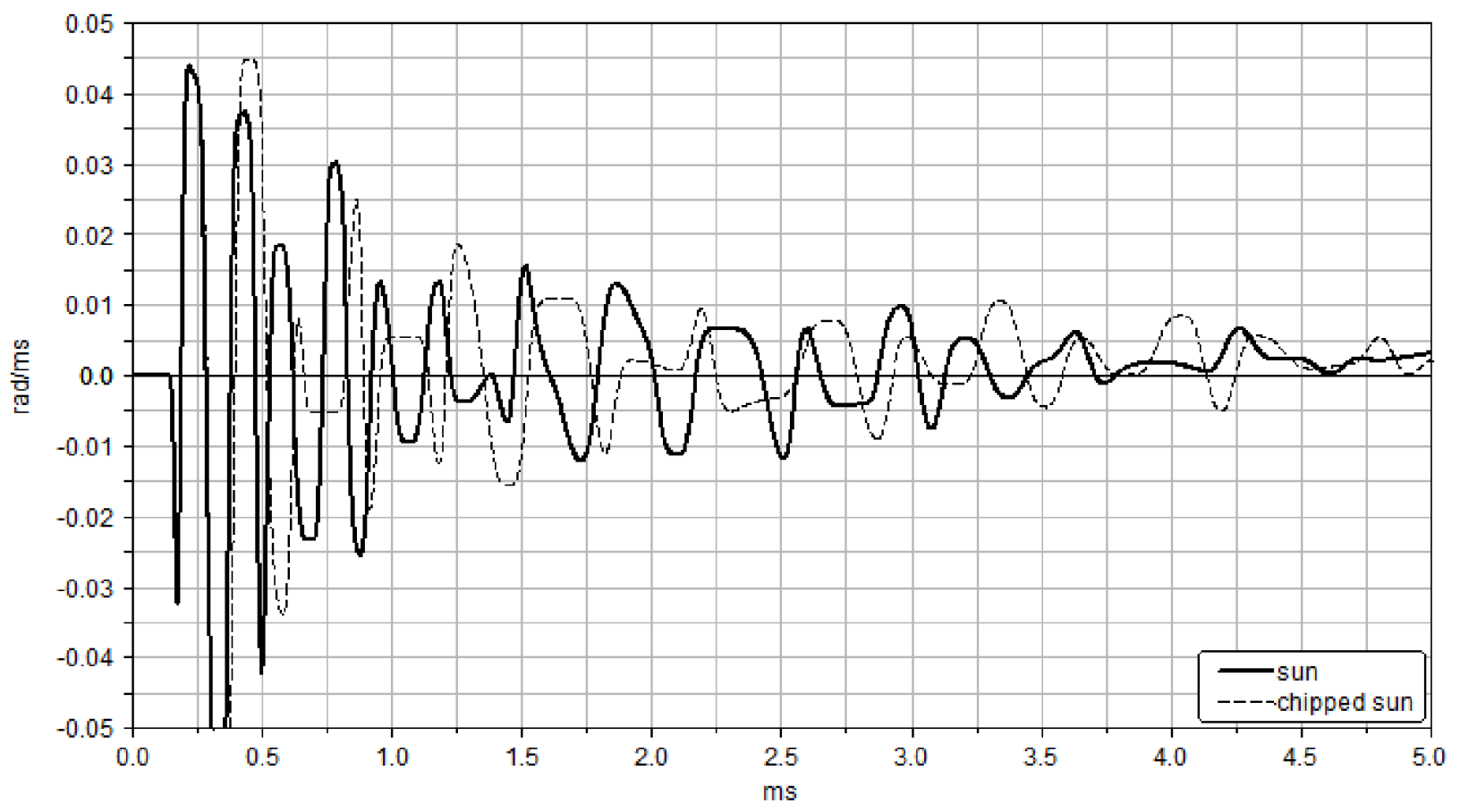

Fig.12 Sun angular velocity vs. time, when the torque $T_{1}=70.518 \mathrm{~N}-\mathrm{m}$ is applied to the sun gear

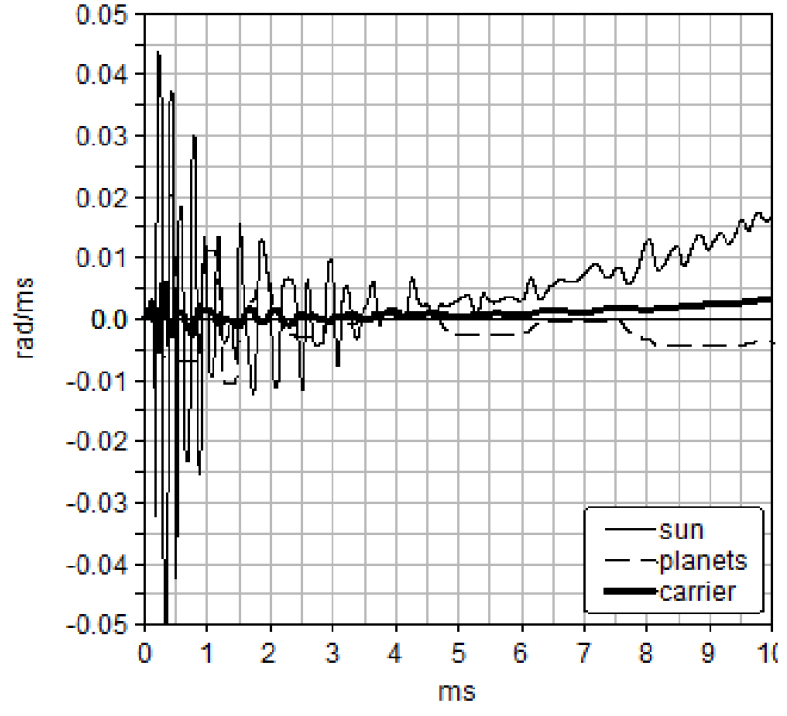

(a) Ring gear fixed, $\mathrm{T}_{1}=70.518 \mathrm{~N}-\mathrm{m}$

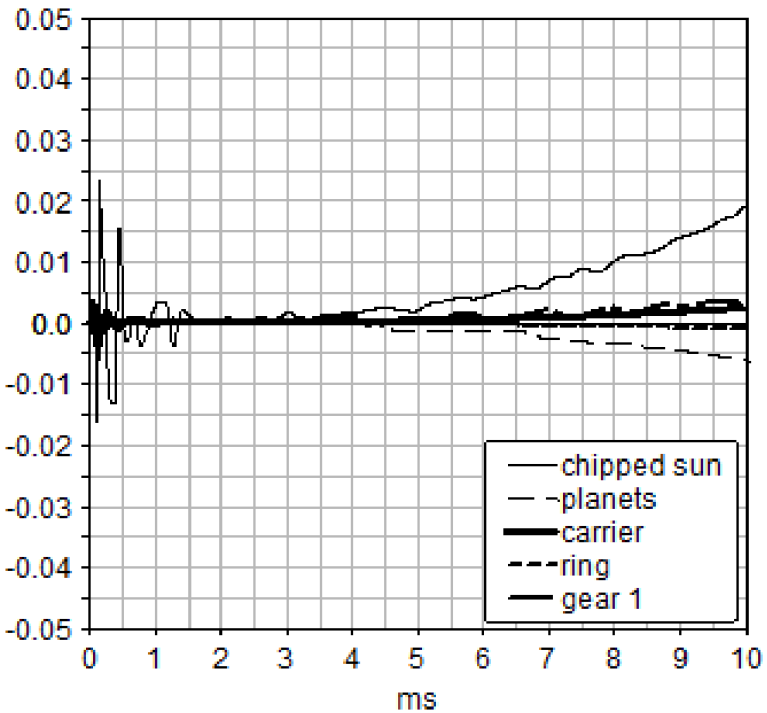

(b) $T_{1}=70.518 \mathrm{~N}-\mathrm{m}, \mathrm{T}_{2}=67.935 \mathrm{~N}-\mathrm{m}$

Fig.13 Angular velocity with applied step torques 


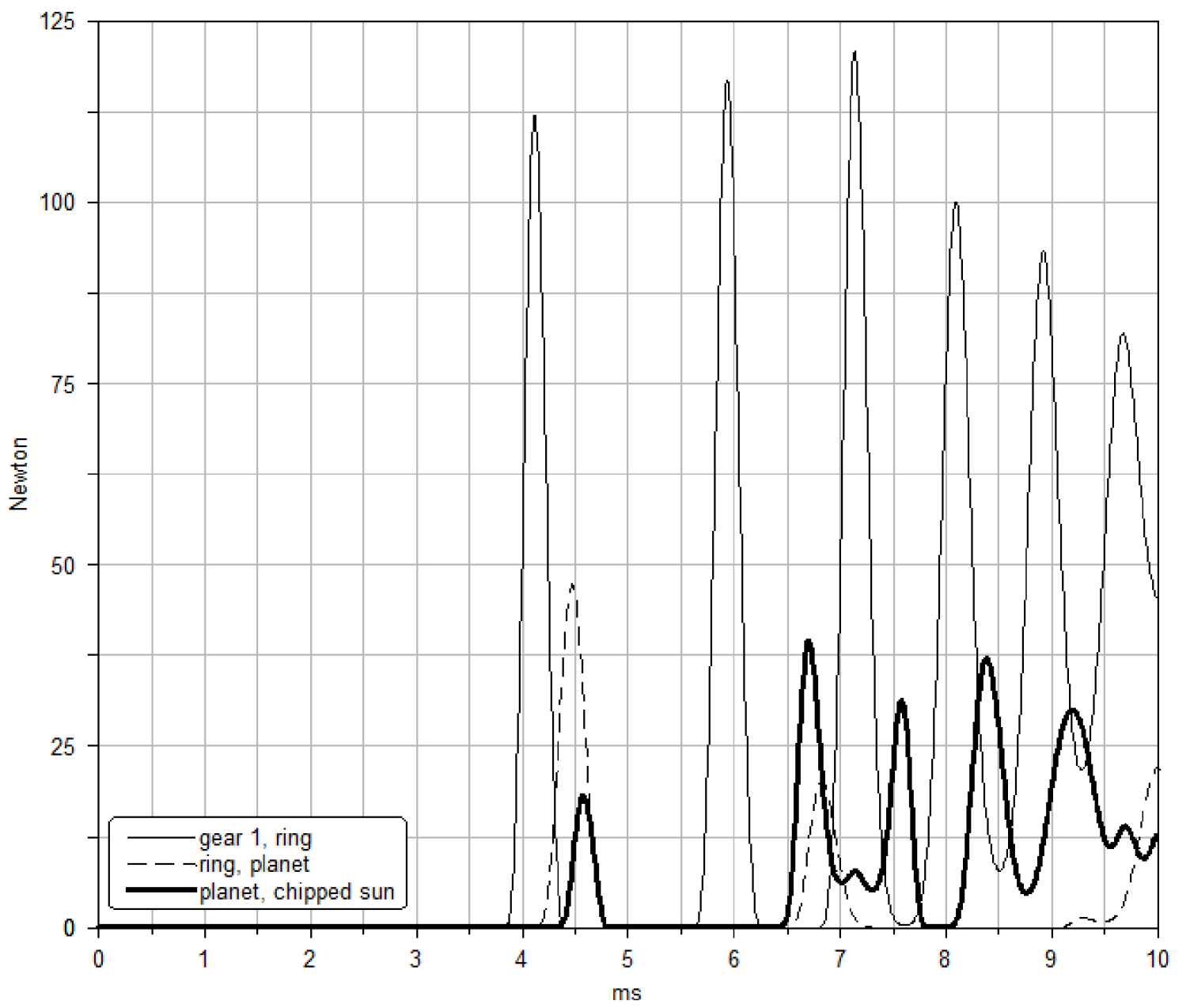

Fig.14 Angular velocity when torque $T_{1}=70.518 \mathrm{~N}$-m is applied to the sun gear and $T_{2}=67.935 \mathrm{~N}$-m is applied to gear 